\section{COMUNICACIÓN Y MODA EN EL ENTORNO \\ DIGITAL: COMPORTAMIENTO DEL PÚBLICO SEGUIDOR DE BLOGUERAS DE MODA}

COMMUNICATION AND FASHION IN THE DIGITAL ENVIRONMENT: THE BEHAVIOR OF FASHION BLOGGERS' FOLLOWERS

\author{
Laura Esteban Santos \\ Universidad de Valladolid, España \\ laura.estebanooo@gmail.com
}

\author{
Irene García-Medina \\ Glasgow Caledonian University, Reino Unido \\ Irene.Garcia2@gcu.ac.uk
}

\author{
Elena Bellido-Pérez \\ Universidad de Sevilla, España \\ ebellido@us.es
}

Para citar este artículo:

Esteban, L., García-Medina, I. \& Bellido-Pérez, E. (2019). Comunicación y moda en el entorno digital: comportamiento del público seguidor de blogueras de moda. Commons. Revista de Comunicación y Ciudadanía Digital, 8(1), 120-155

http://dx.doi.org/10.25267/COMMON.2019.v8.i1.04

Fecha de recepción: 07/09/2018. Fecha de aceptación: 02/11/2018

\section{Resumen}

En este texto se realiza un estudio del fashion blogger o bloguera de moda y del comportamiento del público español en torno a su figura. Para ello, se han realizado encuestas entre la población femenina, con los objetivos de conocer a las blogueras más influyentes del panorama nacional, identificar las diferencias en el comportamiento entre seguidoras y no seguidoras de blogs de moda, y las diferencias entre las seguidoras de una bloguera u otra. Los resultados revelan la volatilidad del compromiso hacia una determinada bloguera o la necesidad por parte de estas de adaptarse a nuevas plataformas como Instagram.

\section{Palabras clave}

Comunicación, moda, nuevos medios, blog

\begin{abstract}
In this text, a study of the fashion blogger is carried on according to the Spanish customer behaviour related to this figure. In order to do that, surveys have been conducted between female population, with the aims of knowing the most influencer bloggers of the Spanish context, identifying the differences between the behaviour of the fashion blogs followers and not followers, and the differences between the followers of one blogger and another. Results show the volatility of the compromise with a particular blogger and the need that these bloggers have of adapting themselves to new platforms such as Instagram.
\end{abstract}

\section{Keywords}

Communication, fashion, new media, blog 


\section{Introducción}

Las nuevas tecnologías han revolucionado la comunicación de manera vertiginosa en los últimos años, creando nuevas e interesantes parcelas de actuación para los distintos sectores sociales y económicos, siendo el sector moda uno de los que más cambios ha experimentado. Al estudiar la conjunción entre comunicación y moda, hoy en día se hace imprescindible dedicar una especial atención a plataformas digitales como los blogs, que, dominados por influencers ${ }^{1}$, se han convertido en auténticos prescriptores en la materia. En este sentido, son varias las investigaciones académicas que comenzaron a interesarse tanto por el blog de moda en general (Perthuis, 2016; Wiazewicz \& Zatwarnicka-Madura, 2016; Kraus \& Martins, 2017) como por la figura del fashion blogger en particular (Pihl \& Sandström, 2013; Larraufie \& Sommer, 2015; Lee, 2016).

Además, de la mano de los avances tecnológicos, no solamente ha cambiado la comunicación en las empresas dedicadas a la moda, sino que, con ellas, también lo han hecho los consumidores, quienes, guiados por la interactividad e inmediatez del medio digital, son más exigentes con los productos que compran. Así pues, buscan que una marca cumpla sus expectativas, que sea activa y que interactúe con ellos dejándoles participar lo máximo posible. En este contexto es donde destaca el avance de los blogs de moda como herramienta imprescindible para conseguir esta interactividad. Estos comenzaron como una afición para muchas de las blogueras ${ }^{2}$ que hoy en día han hecho de ello su profesión, pasando a tener una gran influencia tanto sobre los consumidores y consumidoras como sobre las propias marcas.

1. Según Díaz, el marketing influencer "es una nueva rama del marketing en la que el foco se pone en los líderes de opinión como vía para llegar al consumidor final. [...] Los influyentes [...] ofrecen opiniones personalizadas, sabedores de que cuentan con la confianza de su comunidad [...]. Eso es lo que valora el consumidor final: el influencer es una persona normal y corriente, como ellos" (2017). No obstante, este término no es algo novedoso e intrínseco a las redes sociales, sino que Paul Lazarsfeld y otros teóricos ya señalaron a los líderes de opinión como pieza clave en la comunicación mediática, dada su cercanía con el público (Lazarsfeld, Berelson \& Gaudet, 1968).

2. Se utilizará el término en femenino en el presente texto debido a que la mayoría de blogueras de moda que existen son mujeres. 
Los cambios que los blogs de moda han suscitado en el panorama digital han obligado a las empresas de moda a poner en marcha una renovación continua de sus estrategias de comunicación. Para ello, estas empresas deben, en primer lugar, conocer cómo se ha visto y cómo se ve afectado el comportamiento del consumidor por estas plataformas en general y estas influencers en particular. En definitiva, se ha generado un nuevo tipo de público que, para definirlo, tiene que ser estudiado conforme a su comportamiento y actitudes en torno a este nuevo medio y estos nuevos prescriptores de marca. Más allá de ello, el análisis del público seguidor de este tipo de blogs no solo afecta al marketing mix de las empresas de moda, sino que incide directamente en el estudio del comportamiento del consumidor en el entorno digital.

Con el objetivo principal (OP) de analizar el fenómeno de los blogs de moda desde el punto de vista del público de los mismos, se lleva a cabo en el presente texto un estudio basado en el análisis de resultados de encuestas. Dentro de este objetivo principal se han marcado otros tres objetivos secundarios: (OS1) conocer las diferencias entre el público seguidor y no seguidor de blogs de moda en cuanto a la percepción de dichos blogs y de las blogueras de moda, (OS2), identificar a las blogueras de moda más conocidas del panorama nacional; y (OS3) conocer las diferencias en el comportamiento del público en función de la bloguera de moda que siga.

\section{El blog de moda y el fashion blogger como herramientas de comunicación}

La industria de la moda siempre ha necesitado usar los medios adecuados para comunicarse con su público, del que se espera que surja un deseo de compra independientemente de que sus necesidades básicas estén cubiertas. Entre estos medios destacan, por su tradición y por su evolución de la mano del sector, las revistas y los desfiles. Las primeras, con la irrupción de cabeceras como Cosmopolitan (1886) o Vogue (1892), se popularizaron a finales del siglo XIX, 
cumpliendo una función "divulgativa y prescriptora de las tendencias, a través de sus reportajes, editoriales y bodegones de productos" (Díaz Soloaga, 2007: 121-122). Por su parte, los desfiles de moda siguen siendo una de las grandes herramientas de comunicación en el sector de la moda, debido a su idoneidad a la hora de generar conocimiento de marca y cobertura en los medios, y sobre todo, porque suponen "un impresionante ejercicio de seducción que se cierra con éxito absoluto si la colección se convierte en objeto de deseo, o, aún mejor, de culto" (Mora, 2005: 52).

Pero esta forma de entender la moda y su comunicación comenzó a cambiar con la llegada del denominado street style, el cual ha ido ganando fuerza con el paso de los años hasta el día de hoy, con el auge de la moda de lo anónimo y el cambio de líderes de opinión. Todo ello ha dado lugar, fundamentalmente en la población más joven, a una "migración de lectores y espectadores hacia los nuevos medios digitales" (Celaya, 2008: 70-71). En estos nuevos medios destacan, dentro del sector, las revistas de moda online, que aportan rapidez e introducen la edición diaria, permitiendo una mayor interacción con el púbico; así como los blogs de moda, objeto de estudio de la presente investigación.

El blog en general se puede definir con los rasgos que Díaz y Salaverría extraen de él: es un "espacio de comunicación personal" cuyos contenidos estructurados cronológicamente "abarcan todo tipo de tipología", que permite incluir hiperenlaces y que potencia una interactividad que "aporta un alto valor añadido como elemento dinamizador en el proceso de comunicación” (2003: 296). La gran ventaja del blog es su independencia, aunque, como indica Blood, esta independencia es también su mayor debilidad, puesto que "los blogs, producidos por aficionados, no tienen dicho código [código ético presente en el periodismo], y los bloggers personales casi parecen estar orgullosos de esto" (2002: 116). 
Con la creación de los primeros blogs de estilo personal apareció el primer blog de moda en 2005, seguido por otros muchos (Ruiz, 2013: 3). Como comentan Mortara y Roberti, estos primeros blogs pertenecían a personas aficionadas a la moda, no a trabajadores de la industria, de ahí su carácter amateur (2016: 89). Fue tres años más tarde cuando este fenómeno empezó a cobrar relevancia, produciéndose su desarrollo pleno, en el caso concreto de España, entre los años 2009 y 2010 (Ruiz, 2013: 3). Este desarrollo vivió su momento cumbre con la aparición de las blogueras en los grandes desfiles de moda ${ }^{3}$. Consecuentemente, a partir del 2010 "la discusión en torno a la legitimidad de los blogueros de moda se ha convertido en un tema de reportajes periodísticos, particularmente durante la semana de moda, lo cual indica [...] la creciente influencia de los blogueros" ${ }^{\prime 4}$ (Titton, 2015: 202).

Con el paso del tiempo, los blogs de moda se han ido profesionalizando, creando una sinergia compacta entre el autor del blog, las marcas y la comunidad online. Así, la industria de la moda vio en el fashion blogger una fuente de ingresos y, al ser conscientes las blogueras de ello; "el gran profesionalismo en la aproximación de estos blogueros al sistema de la moda posibilitó que se convirtieran en líderes de opinión capaces de orientar gustos y elecciones compartiendo su estilo personal" 5 (Mortara \& Roberti, 2016: 90). De esta manera, como afirma Parga, "Los bloggers de moda rompieron la jerarquía tradicional de la moda", democratizándola (2015).

3. En este sentido, la Mercedes-Benz Madrid Fashion Week del año 2010 se popularizó en los medios como la "Cibeles de los bloggers" (Ruiz, 2013: 3).

4. Traducción de los autores (TdA).

5. TdA. 
En esta profesionalización destaca la figura del prosumidor ${ }^{6}$, que en la bloguera de moda encuentra un ejemplo clave: esta bloguera es una persona que consume productos de la industria de la moda y produce y reproduce tendencias para su público seguidor, como consecuencia de su rol de líder de opinión o influencer. De esta práctica normalizada en el entorno digital surge el concepto de fashion prosumption ("prosumo de moda"), concepto intrínseco, como apuntan Strähle y Grünewald, a los blogs y los fashion bloggers:

\begin{abstract}
A través de la práctica del blogging, los bloggers forman parte de la creación de la moda influyendo en la diseminación de nuevas tendencias dentro del sistema de la moda. Así, los consumidores son capaces de ejercer un alto grado de influencia y actuar como emprendedores institucionales, involucrándose en actividades de prosumo [...]. Los consumidores y prosumidores cumplen entonces una función clave creando nuevos canales para el marketing de la moda ${ }^{7}$. (2017: 96)
\end{abstract}

Con estos rasgos, los blogs han despertado el interés de las empresas. Sin embargo, como afirman Uzunoglu y Kip, las características de las redes sociales, con una comunicación no jerárquica, dificultan a la empresa dirigir la conversación (2017: 594). La ventaja de utilizar estas plataformas está en que, aunque las marcas no puedan intervenir directamente en esa conversación digital, tienen la oportunidad de influenciarla utilizando a las blogueras como herramienta (Uzunoglu \& Kip, 2017: 594).

Por ello, en este contexto de éxito de los blogs, tanto las propias marcas como las revistas de moda se han visto obligadas a reaccionar. Las primeras, colaborando con las blogueras de moda, y las segundas, incluyendo en su versión digital una sección de blogs, los cuales están protagonizados en su mayoría por mujeres famosas del panorama nacional, como es el caso de Tras la pista de Paula, blog de

6. El "prosumidor" es una figura que surge de unir los roles de "productor" y "consumidor". El concepto ya fue introducido por Toffler en 1980, en su obra La tercera ola (1980), donde describe una fase actual en la que "la sociedad vive vinculada a la electrónica, los medios de comunicación se desmasifican y las personas producen gran parte de su propio consumo" (Andreu-Sánchez \& Martín-Pascual, 2014: 133). Poco tiempo después, este concepto fue aplicado al campo del marketing por Kotler (1986).

7. TdA. 
Paula Echevarría para la revista Elle. Por lo tanto, se puede afirmar que a día de hoy el mundo de los blogs se ha convertido en un negocio que cuenta con diferentes vertientes: es un negocio para la marca, para las agencias intermediarias y para la fashion blogger.

En relación al negocio desde el punto de vista de la marca, las empresas utilizan la fama de las blogueras para promocionar sus productos, y lo hacen a través de colaboraciones para las campañas, como embajadores digitales, es decir, como imagen online de la marca. Tal es el caso de la bloguera Dulceida (Aida Domenech) embajadora digital de la firma Rimmel London e imagen de marca de algunas campañas de Tezenis.

En cuanto a las agencias intermediarias, existen agencias de representación de blogueras, encargadas de llevar a cabo las acciones de representación social, búsqueda de colaboraciones de marcas, consultoría, acuerdos para la participación en eventos especiales, pactos de entrevistas, etc. La primera de ellas fue Okiko Talents, representante, por ejemplo, de Gala González, a la que después siguieron otras como InfluenZia (Escolar, 2014). En el caso de Dulceida, por ejemplo, fue su propia madre la que decidió dejar el trabajo que ejercía y crear DulceWeekend S.L., esto es, la agencia de representación de su hija (Castillo, 2017).

Pero quizá la vertiente más llamativa entre la población sea el negocio de los blogs desde el punto de vista de la fashion blogger. Algunas blogueras de moda han conseguido hacer de su afición por la moda un negocio del que vivir, en base a su gran número de seguidores tanto en el blog como en las redes sociales. Fuentes del sector ofrecían datos en 2017 respecto a lo que cobraba Dulceida: entre $1.300 €$ y $3.000 €$ cada vez que menciona una marca en Instagram, 4.000€ por asistir a un evento y hacerse una foto y $6.000 €$ si pincha música en dicho evento (Castillo, 2017). "Se le paga eso porque es rentable. La marca obtendrá beneficios", afirma esta fuente cercana a la bloguera (Castillo, 2017), lo que redunda en la bidireccionalidad del beneficio. Respecto a lo que sucede fuera de España, la consultora Captiv8 reveló que en Estados Unidos estas influencers de moda cobran "187.500 dólares por post en YouTube, 75.000 en Instagram y 
30.000 en Twitter si tienen entre tres y siete millones de seguidores" (Ovelar, 2017). Pero aparte de los ingresos monetarios, otra gran parte de las ganancias viene dada por los goody bags, es decir, "paquetes regalo con productos de lujo, viajes, pases para spas entre otros, con un valor equiparable al impuesto por la agencia representante" (Díaz Soloaga, 2007: 155), o simplemente prendas de ropa o productos de belleza para que los mencionen en su blog y redes sociales.

Ligado a este último punto, podemos recuperar la identificación que realiza Celaya de "un amplio grupo de personas que participan activamente en la web social con el objetivo de convertirse en un punto de referencia [...]. Algunos interpretan este tipo de participación "interesada" como una forma de alimentar el 'ego' personal" (2008: 25). De ahí ha surgido el término egoblog con el que nos referimos a los blog personales donde el protagonista es el centro de atención del mismo (Ramos-Serrano \& Jiménez-Marín, 2014). Este puede ser de cualquier temática, pero en el mundo de la moda han adquirido una poderosa relevancia, donde estos egobloggers se han convertido en verdaderos fenómenos mediáticos.

Así, entre las egobloggers españolas más influyentes encontramos a Alexandra Pereira con su blog Lovely Pepa; Aida Domenech con Dulceida; Gala González con Amlul; Natalia Cabezas con Trendy Taste o Sara Escudero con Collage Vintange. En el sector masculino, aunque minoritario, destaca en España Pelayo Díaz, con su blog Kate Loves Me. Algunos de estos egobloggers se han convertido en auténticos gurús de la moda. Tanto es así que, como opina Parga, "si el éxito de esta figura se debió a que representaban versiones alcanzables de las celebrities convencionales, la situación ha dado la vuelta: ahora forman parte del sistema" (2015).

Lo cierto es que en el paso desde la confianza original que el público depositaba en estas figuras como modelos de consumo hasta su categoría de influencers, existen varios matices, de los que se pueden resaltar dos. El primero de ellos es el relacionado con la legalidad: siendo un territorio que continuamente se está explorando, aún no existe una regulación estricta para diferenciar las compras 
por buycott ${ }^{8}$ de la mera publicidad en los blogs o en los perfiles de Instagram de estas figuras (Childers, Lemon \& Hoy, 2018; Gómez, 2018). El segundo es el que la presente investigación se propone estudiar: el comportamiento del público en torno a una figura cuya entrada en el sistema mediático la puede convertir en menos personal y creíble, rasgos ambos por los que fue en primer lugar referencia para la población.

En cuanto al medio de comunicación usado, en la actualidad cobra fuerza la pregunta de si estamos asistiendo al fin de los blogs con la conversión de estas egobloggers en instagramers. Instagram es un medio más rápido, cómodo, interactivo y visual que el blog. Las estadísticas de Google avalan la tendencia a la preferencia de esta red por parte del público. Así, comparando las búsquedas del blog y del Instagram de una de las blogueras más famosas de España (gráfico 1), Dulceida, se aprecia la creciente importancia de la red Instagram:

\section{Gráfico 1}

Comparación entre las búsquedas "Dulceida blog" y "Dulceida Instagram" entre agosto del 2014 y agosto del 2017

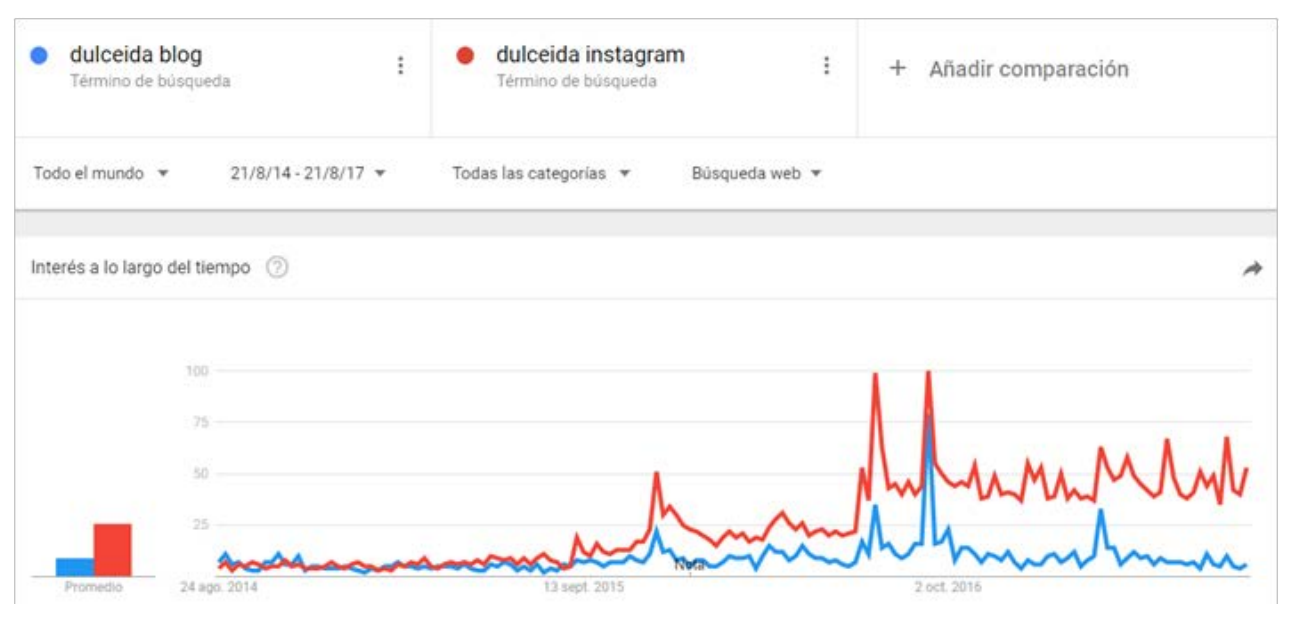

Fuente: https://trends.google.es

8. Buycott es un término creado por Monroe Friedman y referido al consumo político: la compra de productos o servicios a una determinada compañía con el fin de beneficiarla dada la identificación del consumidor con los valores de marca (1996). 
Pero lo cierto es que las blogueras presentes en esta red suelen dejar indicados los enlaces a su propio blog, donde se concentra toda su personalidad ${ }^{9}$. Además, puesto que Instagram se ha adueñado de la básica fotografía con un determinado look, los blogs de moda comienzan a renovarse con otro tipo de contenidos: "Triunfan los posts sobre decoración, salud y bienestar, dietas, running, cocina... Abren canales de YouTube y prueban con tutoriales de belleza" (Parga, 2015). Al fin y al cabo, su presencia y fuerza en otras redes sociales no sustituye el hecho de que estas egobloggers nacieron en un blog de moda.

\section{Metodología}

Para alcanzar los objetivos propuestos, se ha utilizado una metodología cuantitativa, usando la encuesta como técnica de recogida de datos, seguida de un análisis cuantitativo de las respuestas. Al cuestionario ${ }^{10}$, basado en su mayoría en preguntas cerradas, se le dio difusión por redes sociales entre marzo y mayo del 2016. Se publicó en Facebook, Twitter y LinkedIn en perfiles personales, alentando a que fuese compartido entre los contactos para crear una difusión en red. Se buscaba exclusivamente la población de mujeres residentes en España, al ser el sector femenino el seguidor mayoritario de los blogs de moda (Martín \& Martínez, 2017). Finalmente, a pesar de haber previsto una muestra de 200 mujeres, se alcanzó a 133, esto es: respondió el $66,5 \%$ de la muestra deseada. Aun no habiendo logrado el objetivo, se consideró que era una muestra lo suficientemente amplia como para realizar una aproximación a la realidad que se deseaba investigar.

9. En este sentido es significativo que fashion bloggers como Alexandra se sigan considerando blogueras en 2017 (Ovelar, 2017).

10. Ver Anexo. 
Respecto a los diferentes grupos de edad participantes, observamos que el grupo de mayor presencia es el de entre 16 y 30 años, ya que representan el $90 \%$ de la muestra. El siguiente grupo comprende las edades de entre 31 y 50 años, con un $9 \%$, y en último lugar se sitúa el segmento de más de 50 años, que representa tan solo un $1 \%$ de la muestra. La composición de la muestra en cuanto a la edad es bastante desigual, lo cual puede deberse a que las personas que más acceden a las redes sociales se sitúan dentro de ese primer segmento.

De este modo, los objetivos secundarios se abordan en distintas baterías de preguntas, a saber:

- OS1: Conocer las diferencias entre el público seguidor y no seguidor de blogs de moda en cuanto a la percepción de dichos blogs y de las blogueras de moda. Desde el inicio se crean dos rutas de preguntas similares: una destinada a las seguidoras de blogs de moda y otra destinada a las no seguidoras. El contenido de las preguntas es esencialmente el mismo, sin embargo, están formuladas de distinto modo para adaptarse a la persona encuestada. Por ejemplo, si la pregunta 3 para las seguidoras de moda es "cuando visitas un blog de moda, ¿en qué grado prefieres cada uno de los siguientes tipos de publicaciones?", para las no seguidoras será: "En caso de que decidieras seguir algún blog de moda, ¿cuál crees que sería tu grado de preferencia para cada uno de los siguientes tipos de publicaciones?".

- OS2: Identificar a las blogueras de moda más conocidas del panorama nacional. Este objetivo se cumplimentó en la segunda pregunta (2A) del cuestionario, donde se preguntaba por algún bloguero o bloguera de moda que la persona encuestada conociese o siguiese en ese momento. 
- OS3: Conocer las diferencias en el comportamiento del público en función de la bloguera de moda que siga. Realizando un cruce de variables, se estudió si la persona que sigue a una determinada bloguera varía en su comportamiento respecto a las encuestadas que siguen a una bloguera diferente. A partir de la pregunta $2 \mathrm{~B}$, el cuestionario se orientaba a recoger los motivos por los que las usuarias seguían o conocían a las blogueras, así como sus intereses, sus deseos y su comportamiento en el momento de visitar el blog.

\section{Resultados y discusión}

A continuación analizaremos los resultados de la encuesta desglosando las preguntas realizadas.

- Pregunta 1: ¿Sigues algún blog de moda habitualmente?

En primer lugar, se le preguntaba al encuestado si seguía habitualmente algún blog de moda, dividiendo de este modo la muestra en dos grupos: seguidoras y no seguidoras de blogs de moda. Así, de las mujeres encuestadas, un 53\% seguía algún blog de moda habitualmente, mientras que el $47 \%$ restante decía no seguir ninguno. Con esta pregunta se ha podido utilizar la variable "seguir habitualmente un blog de moda" para realizar un cruce analítico con otros resultados, cumpliendo el primer objetivo secundario (OS1) propuesto. 
- Pregunta 2A: (Seguidoras) Indica un bloguero/a de moda que conozcas y sigas en la actualidad / (No seguidoras) Indica un bloguero/a de moda que conozcas (en el caso de que conozcas alguno)

Una vez caracterizada la población que sigue blogs de moda, se ha abarcado el segundo objetivo secundario (OS2), en el cual se requería conocer quiénes son los blogueros o blogueras más notorios para el público objeto de estudio. Para ello se formuló una pregunta de "notoriedad espontánea" y se analizaron los resultados mediante un análisis de las frecuencias (gráfico 2). En este sentido, las tres blogueras de moda más conocidas son: Dulceida, 1sillaparamibolso y LovelyPepa ${ }^{11}$, acumulando entre las tres 53 menciones de las 100 recogidas (ya que 33 personas no dieron ningún nombre), es decir, un 53\%. Cabe destacar que estas tres blogueras pertenecen al grupo de las denominadas egobloggers, mientras que en cuarto lugar, con 10 menciones, encontramos a Paula Echevarría, perteneciente al grupo de las famosas con blog de moda. En cuanto al resto de menciones, se puede destacar que se han nombrado a otros 27 blogueros de moda, pero la mayoría en solo una o dos ocasiones.

\section{Gráfico 2}

Histograma sobre el bloguero/a más conocido

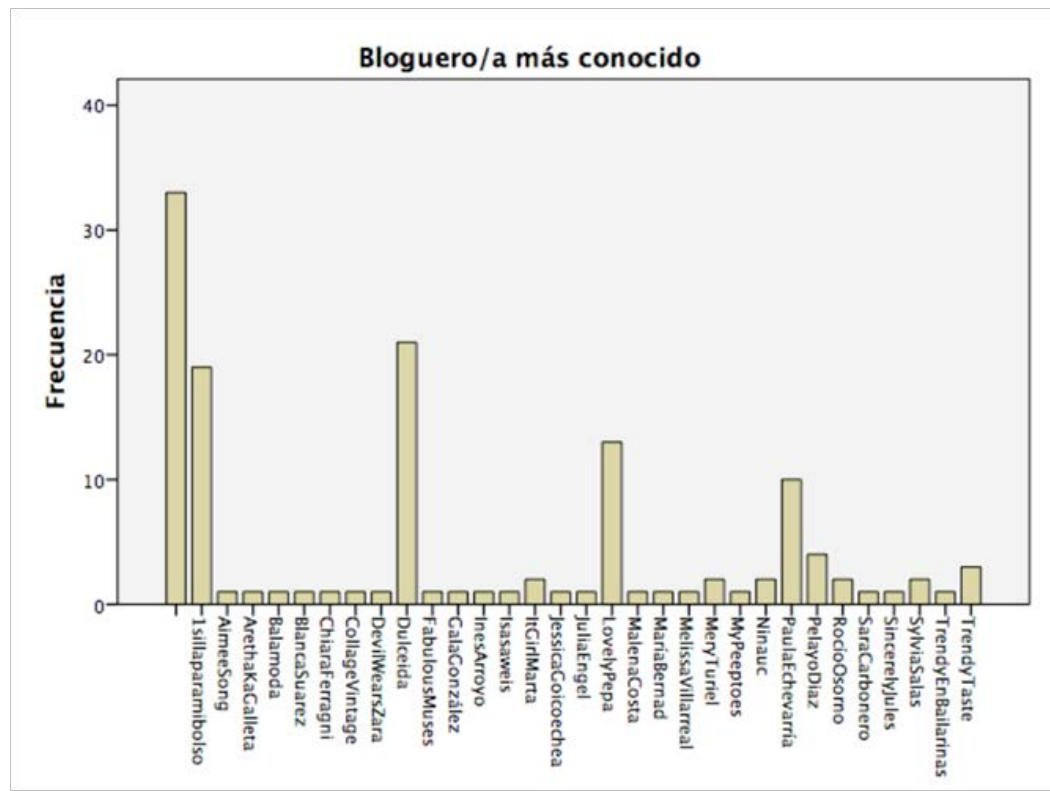

Fuente: elaboración propia

11. Sus respectivos blogs son: http://www.dulceida.com/, http://1 sillaparamibolsoblog.es/ y http://lovelypepa.com/. 
Posteriormente, con el fin de comprobar silas blogueras másconocidas cambiaban al analizar solo la parte de la muestra que sí que sigue habitualmente algún blog de moda, se ha procedido a realizar el análisis seleccionando únicamente estos casos y observando que el resultado es el mismo: las tres blogueras más conocidas son de nuevo Dulceida, 1sillaparamibolso y LovelyPepa, manteniendo también el mismo orden.

- Pregunta 2B: (Seguidoras) Es el bloguero/a que más sigo porque...

Para conocer las razones por las que las mujeres deciden seguir a una determinada bloguera, se incluyó una pregunta con diferentes motivos. En ella, las encuestadas debían indicar, mediante una escala de Likert desde "totalmente en desacuerdo" hasta "totalmente de acuerdo", el nivel de influencia que tenía cada uno de dichos motivos a la hora de seguir a su bloguera favorita.

En el análisis general de las frecuencias de respuesta se observó que los principales motivos son el gusto por su estilo, el gusto por sus fotos y que la bloguera parece conocedora de los temas que trata. Por otro lado, los motivos que menos influyen son el interés por la vida de la bloguera y el hecho de que también sea seguida por los amigos o amigas de la persona encuestada.

Tras esta observación, se realizaron tres análisis ANOVA para saber si los motivos eran significativamente diferentes en función de cada una de las tres blogueras más conocidas (comenzando así a cumplir el OS3, que marcaba conocer las diferencias en el comportamiento del público en función de la bloguera de moda que siga). En este caso, no se ha encontrado ninguna diferencia significativa, excepto en el caso de Dulceida, donde se rechaza la hipótesis nula en la variable "Es el bloguero/a que más sigo porque es el más entretenido/a” con un p-valor de 0,014. De ahí se puede afirmar que las seguidoras de esta bloguera se sienten más motivadas a seguirla porque consideran que es más entretenida que el resto. 
- Pregunta 2C: (Seguidoras) Le/la sigo en... / (No seguidoras) Le/la conozco por...

Con esta pregunta se pretende estudiar si las blogueras más conocidas lo son realmente por su blog o si se debe a su actividad en las redes sociales, fundamentalmente Instagram, como ya se apuntaba en el marco teórico. A su vez, observaremos si existen diferencias en función de si la persona es seguidora habitual de blogs de moda o no. Así pues, se ha llevado a cabo un análisis de contingencia, el cual ha arrojado un p-valor del 0,000 en la prueba de la chicuadrado, rechazando por tanto la hipótesis nula y haciendo evidentes las diferencias significativas en función de la variable comentada anteriormente.

En primer lugar, haciendo referencia al total de la muestra, se puede comprobar (tabla I) cómo el mayor porcentaje corresponde a la opción de conocer/seguir a la bloguera a través de su Instagram. Si se centra la atención en el grupo de personas que sí siguen blogs de moda, la opción preferida es la de seguir a la bloguera tanto en su blog como en Instagram (43,7\%). En el caso de los que no siguen blogs habitualmente, la opción predominante es la de no conocerle por ninguna de las plataformas $(43,5 \%)$, estando en segundo lugar la opción de conocerle por Instagram $^{12}(35,5 \%)$.

12. A las no seguidoras de blogs se les preguntó exclusivamente a través de qué plataforma conocían al bloguero/a de moda. Por ello, en este dato cabe la posibilidad de que las personas que no sigan blogs de moda pero que conozcan a través de Instagram a blogueros/as de moda, también los sigan en esta red social. 
Tabla I

Análisis de contingencia entre seguir blogs y plataformas en la que conoces a tu bloguera preferida

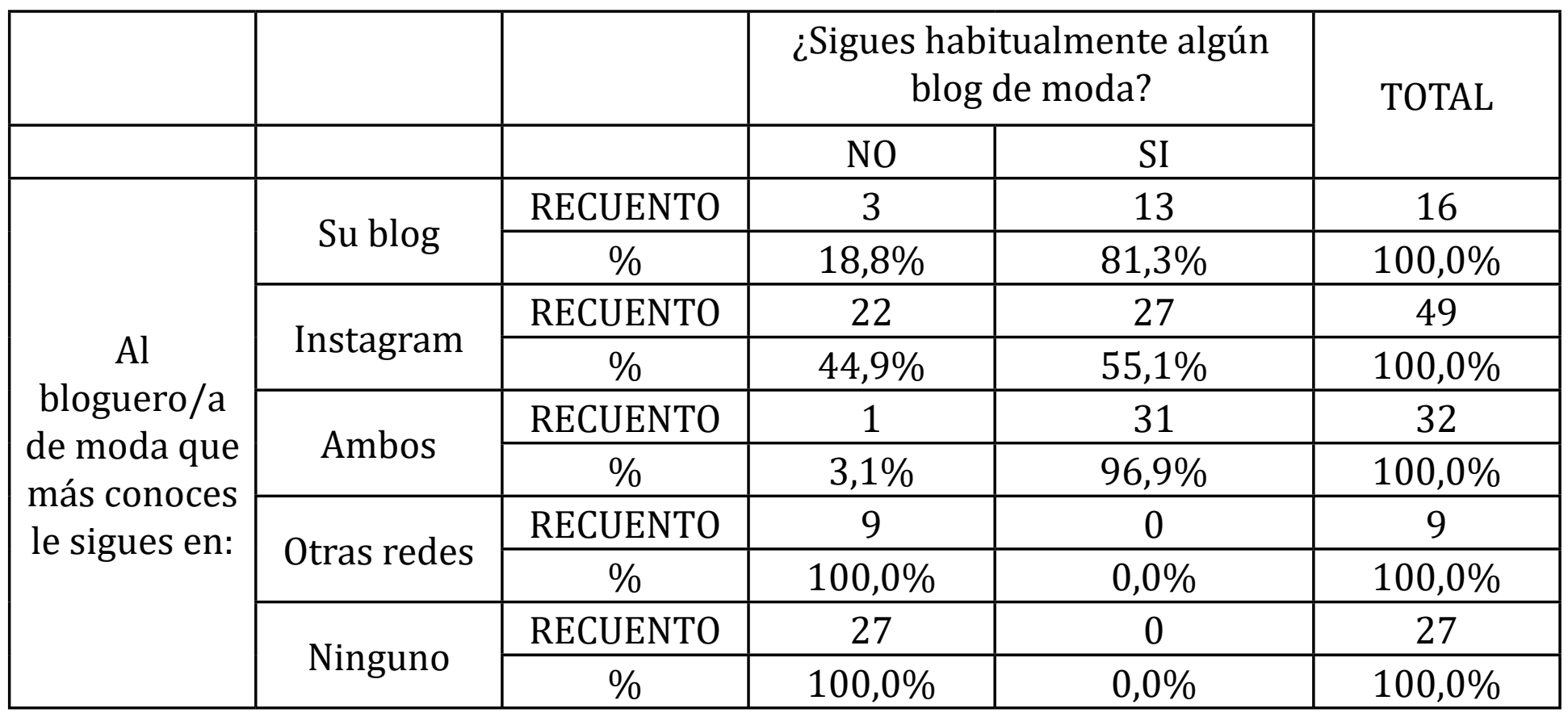

Fuente: elaboración propia

Tras este análisis general se ha procedido a comprobar en qué plataforma son más seguidas las tres blogueras mencionadas anteriormente, y para ello se ha realizado nuevamente un análisis de las frecuencias. En el caso de Dulceida y 1sillaparamibolso, se ha observado que son más conocidasy seguidas en Instagram entre la muestra. Llama la atención que nadie ha declarado conocer o seguir a Dulceida exclusivamente en su blog, lo que demuestra la gran importancia que están adquiriendo redes sociales como Instagram para las blogueras. Respecto a LovelyPepa, la opción más escogida ha sido la de conocerla o seguirla tanto en el blog como en Instagram ${ }^{13}$.

13. Los seguidores de Dulceida (@dulceida) en Instagram son 2.500.000, los de 1sillaparamibolso (@silvianavarrocollection) 357.000, y los de LoveyPepa (@lovelypepa) 1.700.000. (Perfiles consultados el 16 de noviembre del 2018). 
- Pregunta 2D: (Seguidoras) ¿Con qué frecuencia visitas su blog?

Otro de los aspectos a conocer es la frecuencia con la que las seguidoras de una determinada bloguera visitan su blog, derivando así en un determinado grado de interés y atención por sus contenidos. Para ello comenzamos haciendo un estudio de las frecuencias obtenidas en el cuestionario (gráfico 3), donde se observa que la opción más escogida ha sido la de visitarlo al menos una vez por semana $(77,5 \%)$, seguido de la opción "de forma muy esporádica" y, por último, "todos los días".

\section{Gráfico 3}

Distribución de las seguidoras de blogs en función de la frecuencia con la que visitan blogs de moda

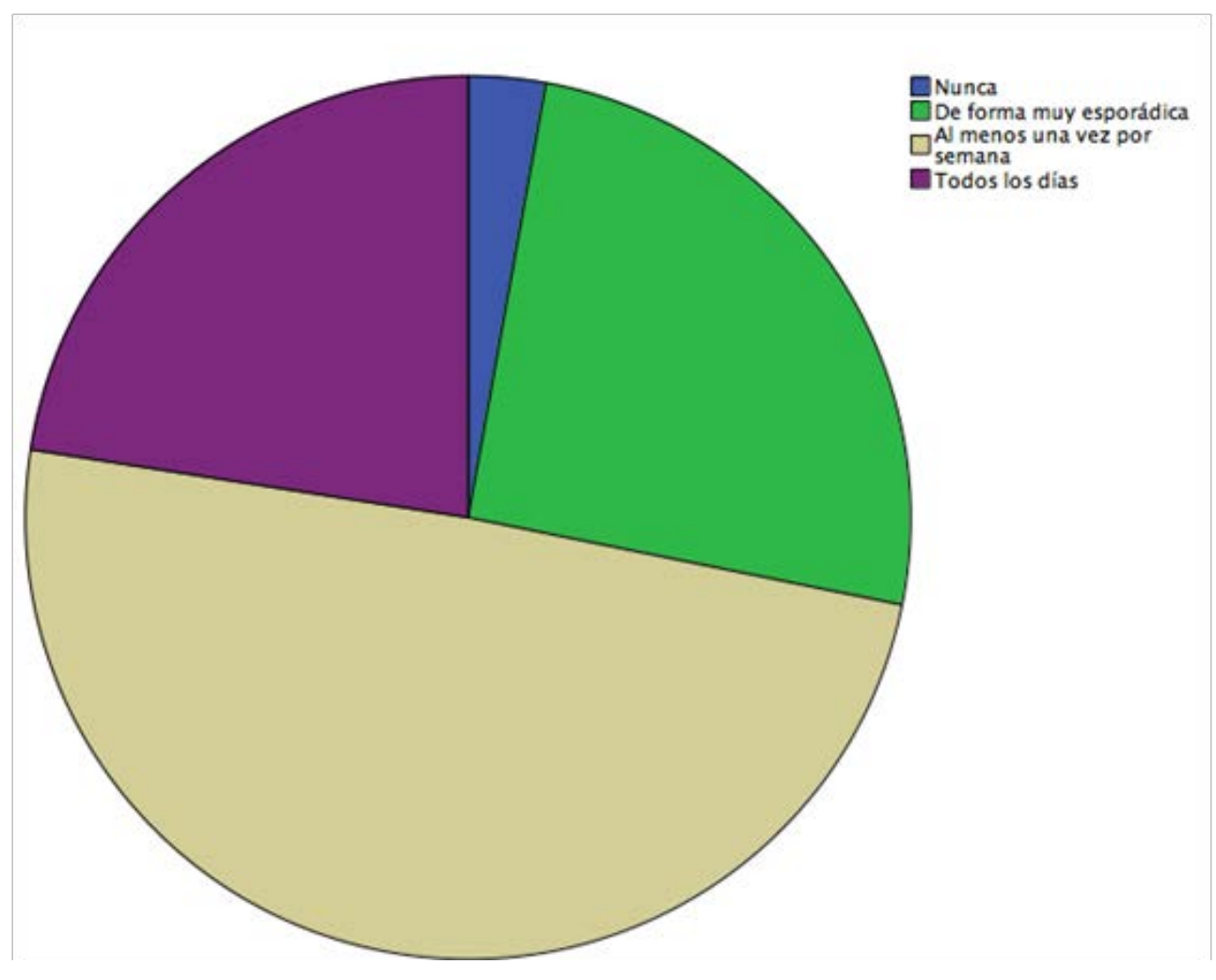

Fuente: elaboración propia 
Una vez extraída la frecuencia entre todas las seguidoras habituales de blogs de moda, se ha procedido a analizar esta frecuencia entre las seguidoras de las tres blogueras más conocidas. Como resultado, se ha obtenido que las encuestadas que visitan el blog con mayor frecuencia son las seguidoras de 1sillaparamibolso, seguidas de las de LovelyPepa y en último lugar las de Dulceida, ya que, como ya se ha visto anteriormente, esta es seguida mayormente a través de Instagram.

- Pregunta 2E: (Seguidoras) Marca la opción que más se ajuste a ti en las siguientes cuestiones sobre el seguimiento de tu bloguero/a favorito/a

Para conocer el grado de lealtad de las mujeres seguidoras de blogs de moda hacia su bloguera favorita se realizó esta pregunta, en la que las encuestadas debían valorar en una escala de Likert desde "totalmente en desacuerdo" hasta "totalmente de acuerdo" cada una de las opciones. El análisis de frecuencias resultó del siguiente modo (tabla II):

Tabla II

Medias de acciones que miden la lealtad a un bloguero/a

\begin{tabular}{|l|c|c|c|}
\hline & $\mathrm{N}$ & Media & Desviación \\
\hline Sigo en las RRSS a mi bloguero/a favorito/a & 71 & 3,68 & 1,402 \\
\hline $\begin{array}{l}\text { Creo que mi bloguero/a favorito/a se está } \\
\text { convirtiendo en un negocio }\end{array}$ & 71 & 3,27 & 1,492 \\
\hline $\begin{array}{l}\text { No me pierdo ninguna publicación de mi } \\
\text { bloguero/a favorito/a }\end{array}$ & 71 & 2,79 & 1,403 \\
\hline Recomiendo el blog de mi bloguero/a favorito/a & 71 & 2,75 & 1,328 \\
\hline Imito el estilo de mi bloguero/a favorito/a & 71 & 2,70 & 1,101 \\
\hline $\begin{array}{l}\text { Participo en los sorteos de mi bloguero/a } \\
\text { favorito/a }\end{array}$ & 71 & 2,65 & 1,455 \\
\hline $\begin{array}{l}\text { Compro los productos que recomienda mi } \\
\text { bloguero/a favorito/a }\end{array}$ & 71 & 2,61 & 1,236 \\
\hline
\end{tabular}




\begin{tabular}{|l|c|c|c|}
\hline $\begin{array}{l}\text { Creo que mi bloguero/a favorito/a está perdiendo } \\
\text { parte de su esencia }\end{array}$ & 71 & 2,14 & 1,279 \\
\hline $\begin{array}{l}\text { Me considero miembro de la comunidad de mi } \\
\text { bloguero/a favorito/a }\end{array}$ & 71 & 2,03 & 1,320 \\
\hline $\begin{array}{l}\text { Comento en las publicaciones de mi bloguero/a } \\
\text { favorito/a }\end{array}$ & 71 & 1,69 & 1,050 \\
\hline $\begin{array}{l}\text { Participo en foros relacionados sobre mi } \\
\text { bloguero/a favorito/a }\end{array}$ & 71 & 1,61 & 1,049 \\
\hline $\begin{array}{l}\text { Pertenezco al club de fans de mi bloguero/a } \\
\text { favorito/a }\end{array}$ & 71 & 1,42 &, 936 \\
\hline N válido (por lista) & 71 & & \\
\hline
\end{tabular}

Fuente: elaboración propia

Se observa que las opciones mayoritarias son: seguir a la bloguera en sus redes sociales, creer que se está convirtiendo en un negocio, no perderse ninguna publicación, y recomendarle el blog a otras personas. Por otro lado, las opciones con menor media son las relacionadas con la participación activa, como comentar en las publicaciones, participar en foros relacionados o pertenecer a su club de fans.

- Pregunta 3: (Seguidoras) Cuando visitas un blog de moda, ¿en qué grado prefieres cada uno de los siguientes tipos de publicaciones? / (No seguidoras) En caso de que decidieras seguir algún blog de moda, ¿cuál crees que sería tu grado de preferencia para cada uno de los siguientes tipos de publicaciones?

Resultaba conveniente incluir una pregunta que recogiese las frecuencias con las que las encuestadas prefieren cada tipo de publicación (tabla III), sobre todo teniendo en cuenta la creciente diversificación en contenidos presente en los blogs de moda. Para ello, se categorizaban en el cuestionario distintos tipos de publicaciones y las encuestadas debían indicar el interés que cada uno de ellos le suscitaba en una escala de Likert comprendida entre "nada" y "totalmente". 
Tabla III

Medias de preferencia de los diferentes tipos de publicaciones

\begin{tabular}{|l|c|c|c|}
\hline Tipo de publicación & $\mathrm{N}$ & Media & Desviación \\
\hline Post de viajes & 133 & 2,67 & 1,146 \\
\hline Outfit of the day & 133 & 2,63 & 1,190 \\
\hline Post de maquillaje & 133 & 2,29 & 1,171 \\
\hline Post de decoración & 133 & 2,12 & 1,243 \\
\hline DIY & 133 & 1,95 & 1,173 \\
\hline Reviews & 133 & 1,91 & 1,252 \\
\hline Post de sorteos/concursos & 133 & 1,80 & 1,254 \\
\hline Hauls & 133 & 1,70 & 1,243 \\
\hline Colaboraciones con marcas & 133 & 1,53 & 1,070 \\
\hline N válido (por lista) & 133 & & \\
\hline
\end{tabular}

Fuente: elaboración propia

En esta tabla se puede observar que las publicaciones preferidas por las mujeres de la muestra versan sobre viajes, con una media de 2,67 , seguidas de las publicaciones sobre el outfit of the day, en las que la bloguera muestra el estilismo completo del día, facilitando información de las prendas, precios, tiendas en las que encontrar productos similares, etc.; y de las publicaciones sobre maquillaje y decoración. Por el contrario, las publicaciones por las que las encuestadas han mostrado un menor grado de preferencia son los hauls, en los que la bloguera muestra sus últimas compras, y las publicaciones en las que colabora con alguna marca. Este dato, con las publicaciones sobre viajes a la cabeza, reafirma la importancia de la variedad de contenidos en el blog de moda, dada en parte a causa de la migración de las blogueras a Instagram (Parga, 2015), $y$, por ende, siendo una estrategia que potencia o mantiene el valor del blog frente a la red social. 
Una vez identificadas las publicaciones con mayor y menor grado de preferencia, pasamos a diferenciar los resultados entre el grupo de seguidoras y no seguidoras de un blog de moda. Para ello, realizamos un análisis ANOVA de un factor, con el objetivo de comprobar si la media de preferencia es igual entre ambos grupos de mujeres. Al realizar el análisis, se pudo observar que en algunos tipos de publicaciones se rechaza la hipótesis nula al ser su p-valor inferior al 0,05, es decir, que en estos casos el grado de preferencia será significativamente mayor en uno de los dos grupos. Por parte de las mujeres que sí siguen habitualmente blogs de moda, se observa una mayor preferencia por las publicaciones de reviews de productos, en las que se hace una reseña de ellos, por las de outfit of the day, y por los hauls. En el caso de las mujeres que no siguen blogs de moda, se puede observar un mayor grado de preferencia por las publicaciones sobre viajes.

A partir de la información obtenida, se ha procedido a segmentar la muestra para identificar cuáles serían los tipos de publicación dentro del público de las tres blogueras más conocidas y así comprobar si existen diferencias entre ellas:

- Dulceida: su tipo de publicación preferido por las seguidoras encuestadas es el de Outfit of the day. En cuanto a la publicación que menos les gusta, coinciden con el público general en citar las colaboraciones con marcas.

- 1sillaparamibolso: las publicaciones preferidas por sus seguidoras son las de maquillaje, seguidas por las de viajes y estilismo del día. Las publicaciones que menos gustan son, de nuevo, las de colaboraciones con marcas.

- LovelyPepa: sus seguidoras han mostrado un mayor grado de preferencia por las publicaciones de Outfit of the day, y un menor grado por las colaboraciones con marcas.

- Pregunta 4: (Seguidoras) ¿En qué medida crees que te influyen cada uno de los siguientes aspectos a la hora de seguir un blog o no? 
Una de las informaciones más interesantes extraídas del cuestionario es la relativa a la influencia que las seguidoras de blogs creen que pueden ejercer determinados motivos a la hora de seguir un blog de moda. Se ofreció una lista con varios motivos que debían ser valorados en una escala de Likert que abarcaba desde "nada" hasta "totalmente"; posteriormente se trataron los resultados en un análisis de frecuencias. A través de las frecuencias se pudo observar cómo las variables que se auto perciben con mayor influencia son el gusto por los temas tratados, la accesibilidad y la credibilidad, mientras que lo que se cree que menos influye es el entorno social de la encuestada.

- Pregunta 5: (Seguidoras y no seguidoras) A la hora de buscar inspiración para tus compras, ¿consultas blogs de moda?

Para comprobar si existen diferencias significativas cuando se decide consultar un blog de moda buscando información, se ha decidido cruzar esta variable con el seguimiento habitual de blogs de moda. Así, se ha realizado un análisis de contingencia con la variable “isigues habitualmente algún blog de moda?” (tabla IV), observando que las diferencias son significativas, al rechazar la hipótesis nula con un p-valor de 0,000. 


\section{Tabla IV}

Análisis de contingencia entre ser seguidora de blogs y consultar blogs para buscar inspiración

\begin{tabular}{|c|c|c|c|c|c|}
\hline & & & \multicolumn{2}{|c|}{$\begin{array}{c}\text { A la hora de buscar } \\
\text { inspiración para tus compras } \\
\text { ¿consultas blogs de moda? }\end{array}$} & \multirow[t]{2}{*}{ Total } \\
\hline & & & No & Sí & \\
\hline \multirow{4}{*}{$\begin{array}{l}\text { ¿Sigues } \\
\text { habitualmente } \\
\text { algún blog de } \\
\text { moda? }\end{array}$} & \multirow{2}{*}{ No } & Recuento & 52 & 10 & 62 \\
\hline & & $\%$ & $83,9 \%$ & $16,1 \%$ & $100,0 \%$ \\
\hline & \multirow{2}{*}{ Sí } & Recuento & 17 & 54 & 71 \\
\hline & & $\%$ & $23,9 \%$ & $76,1 \%$ & $100,0 \%$ \\
\hline \multirow[t]{2}{*}{ Total } & Recuento & 69 & 64 & 133 & \\
\hline & $\%$ & $51,9 \%$ & $48,1 \%$ & $100,0 \%$ & \\
\hline
\end{tabular}

Fuente: elaboración propia

Tal y como se muestra en la tabla, las que consultan con menos frecuencia blogs de moda a la hora de buscar inspiración son las que no siguen habitualmente este tipo de blogs. Sin embargo, llama la atención que exista un 16,1\% de las que no son seguidoras de blogs de moda que sí consulten dichos blogs para inspirarse en sus compras. También es llamativo que un $23,9 \%$ de las seguidoras habituales de blogs no los consulte cuando busca inspiración.

- Pregunta 6: (Seguidoras) De los productos que se muestran en el blog, ¿cuántos sueles comprar?

Por último, en relación a todo lo tratado respecto a los blogs de moda femeninos, resulta también interesante su repercusión en las ventas de las marcas, por ello se incluyó una pregunta sobre la cantidad de productos mostrados en el blog que suelen adquirir las encuestadas (gráfico 4). 


\section{Gráfico 4}

Distribución de las seguidoras de blogs según la cantidad de productos mostrados en el blog que compran

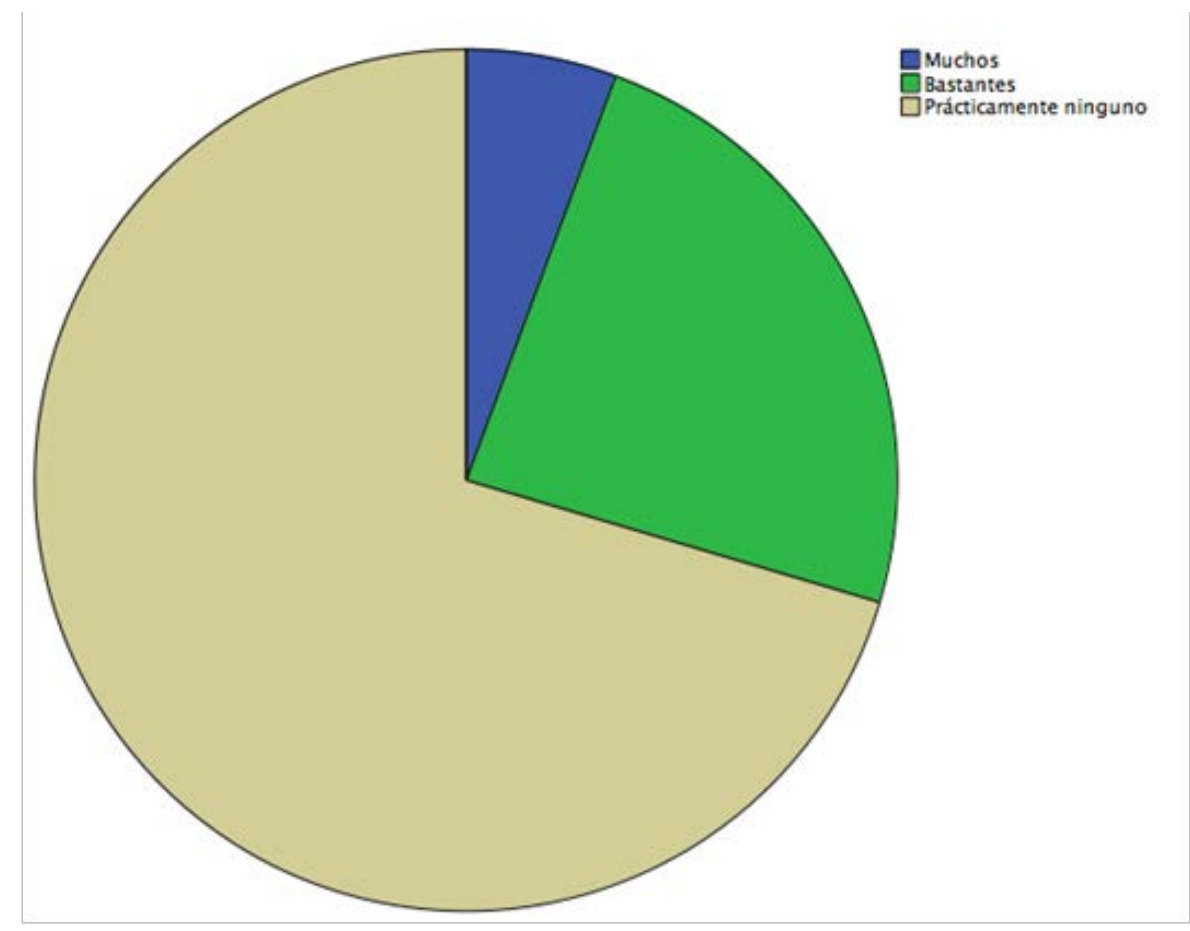

Fuente: elaboración propia

A través del análisis de las frecuencias, se puede observar cómo la mayoría afirma que no compra prácticamente ninguno de los productos que se muestran en el blog $(70,4 \%)$, seguido de la opción de comprar bastantes productos $(23,9 \%)$. En este caso, es interesante comprobar si existen diferencias entre las seguidoras de Dulceida, 1sillaparamibolso y LovelyPepa. No obstante, tras analizar sus respectivas frecuencias y realizar un análisis de contingencia en cada caso, se ha visto que coinciden con lo observado en la muestra general y que, por tanto, no existen diferencias significativas en función de si son seguidoras o no de estas blogueras en concreto. 


\section{Conclusiones}

La realización del presente estudio ha supuesto una aproximación a la relación que mantienen las blogueras de moda con su público en el entorno digital. En este sentido, se parte de la base de que uno de los mayores cambios que ha supuesto el paso del universo offline al online ha sido la dirección del flujo de información: si antes eran las propias marcas de moda o las revistas las que informaban de las novedades, las tendencias o daban consejos de moda y belleza, a día de hoy, a esa comunicación se le ha sumado el usuario en general y el fashion blogger en particular quien, convertido en prosumidor, solicita, participa y da forma a las tendencias del momento a través de diversos productos (los cuales muestra por iniciativa propia en primer lugar y en colaboración con la marca en un siguiente paso).

Así, de manera general, mediante el análisis de los resultados extraídos se puede afirmar que las principales seguidoras de blogs de moda son actualmente mujeres jóvenes que acuden a ellos como fuente de inspiración, prefiriendo los blogs de moda personales o egoblogs. Dentro de este formato de blogs, las publicaciones con mayor acogida son aquellas en las que se muestran los viajes o el estilismo del día, mientras que las menos interesantes para el público son las colaboraciones con marcas. La lealtad de este público seguidor no es muy elevada: se basa en el seguimiento o la recomendación, pero no tanto en la participación activa, presentando una frecuencia de visita al blog de al menos una vez por semana de forma general. Respecto a los motivos por los que se sigue cualquier blog de moda, destacan entre ellos los temas tratados y la accesibilidad; $y$, las razones que suelen llevar a seguir una bloguera en particular son el estilo, la calidad de las fotos y la confianza en la bloguera.

Lo cierto es que las blogueras se han convertido en canales de difusión para la industria de la moda, al revelarse como una herramienta perfecta a la hora de publicitar sus productos. Estos blogs están integrados en su mayoría por 
personas ajenas al mundo de la comunicación, esencialmente mujeres como se ha podido ver en el análisis, pero con un gran interés por el mundo de la moda, lo que les ha llevado a ser verdaderos prescriptores aceptados por la sociedad. Esta realidad camina pareja a otra apuntada por algunos periodistas y manifestada en los resultados del análisis: la sensación de que las blogueras de moda se están convirtiendo en un negocio, que se debe, como opinaba Parga (2015), a que su notoriedad ya les ha hecho entrar a formar parte del sistema de mercado. Esto, junto con la problemática de la publicidad encubierta, puede entrar en conflicto, a la luz de los resultados, con el tercer factor mejor valorado a la hora de seguir un blog: la credibilidad.

De todo ello se deduce que el ascenso y caída de la fama de una bloguera están altamente condicionados por las actitudes y el comportamiento de su público seguidor, capaz de colocar y eliminar egobloggers del panorama digital y hacer que, consecuentemente, las empresas de moda no dejen de buscar a la bloguera más adecuada a su target. Con esta estrecha relación entre un público que convierte a la bloguera de moda en líder de opinión y una empresa que pretende acercarse a este público utilizando a la bloguera, en la actualidad, tener un blog de moda exitoso se considera una actividad profesional, al mismo nivel de otras tantas profesiones surgidas en los últimos años con la aparición de las nuevas tecnologías.

No obstante, debido al propio hecho del rápido avance de las nuevas tecnologías, al aumento del ritmo de vida y a una creciente preferencia por la imagen frente al texto, la población exige una inmediatez en los medios a la que la bloguera de moda debe adaptarse. De ahí que se viva una continua migración de blogueras consolidadas a redes sociales como Instagram, en las que mantienen una incesante actividad, o que surjan directamente instagramers dedicados a la moda, también convertidos en un negocio para las marcas. Los resultados de este estudio han demostrado una clara preferencia por esta red, fundamentalmente visual, frente al blog de moda. Pero ello no significa que el blog de moda en su concepción original esté desapareciendo, sino que su formato está evolucionando conforme a las demandas de la sociedad y de las nuevas tecnologías. 
Por último, resulta necesario mencionar las limitaciones de la presente investigación en cuanto a la población estudiada: al distribuir el cuestionario entre los contactos personales y profesionales puede darse una red muy estrecha de mujeres que han accedido a responder dicho cuestionario. Por ello, los resultados obtenidos, además de no alcanzar el tamaño pretendido, deben observarse como una aproximación al comportamiento de la consumidora del blog de moda, y no como una muestra extrapolable al comportamiento general de la población española. Por este motivo, se está trabajando en la continuación de esta investigación con el objetivo de ampliar la muestra y orientar el cuestionario al comportamiento de los consumidores en torno a los perfiles de las fashion bloggers con más seguidores en Instagram. 


\section{Bibliografía}

- ANDREU-SÁNCHEZ, C. \& MARTÍN-PASCUAL, M. (2014). La educación audiovisual y la creación de prosumidores mediáticos. Estudio de caso. AdComunica, 7, 131-147. doi: 10.6035/2174-0992.2014.7.8

- BLOOD, R. (2002). The Weblog Handbook: Practical Advice on Creating and Maintaining Your Blog. Cambridge: Perseus Publishing.

- CASTILlO, A. (2017, 16 de septiembre). Dulceida: un imperio de dos millones de seguidores en Instagram. El Mundo. Recuperado de: https://www.elmundo. es/loc/famosos/2017/09/16/59bbfd0de2704e8a0b8b45f0.html

- CELAYA, J. (2008). La empresa en la web 2.0. Barcelona: Planeta de Agostini.

- CHILDERS, C. C., LEMON, L. L., \& HOY, M. G. (2018). \#Sponsored \#Ad: Agency Perspective on Influencer Marketing Campaigns. Journal of Current Issues \& Research in Advertising, 1-17. doi:10.1080/10641734.2018.1521113

- DÍAZ, L. (2017). Soy marca. Quiero trabajar con influencers. Influencer marketing. Barcelona: Profit.

- DÍAZ NOCI, J. \& SALAVERRÍA, R. (2003). Manual de redacción ciberperiodística. Barcelona: Ariel.

- DÍAZ SOLOAGA, P. (2007). El valor de la comunicación. Cómo gestionar marcas de moda. Madrid: Cie Inversiones Editoriales Dosset.

- ESCOLAR, M. (2014, 13 de marzo). Blogueros de moda, la profesionalización de un 'hobbie'. Zoom News. Recuperado de https://goo.gl/j3yqEg

- FRIEDMAN, M. (1996). A Positive Approach to Organized Consumer Action: The "Buycott" as Alternative to the Boycott. Journal of Consumer Policy, 19(4) 439-451.

- GÓMEZ NIETO, B. (2018). El influencer: herramienta clave en el contexto digital de la publicidad engañosa. Methaodos. Revista de Ciencias Sociales, 6 (1), 149156. doi: http://dx.doi.org/10.17502/m.rcs.v6i1.212

- KOTLER, P. (1986). The Prosumer Movement: a New Challenge for Marketers. NA - Advances in Consumer Research, 13, 510-513. 
- KRAUS, A. \& MARTINS, N. (2017). On the Street: A Content Analysis of Body Imagery in Streetstyle Fashion Blogs. Journal of Broadcasting and Electronic Media. 61(2), 351-367. doi: 10.1080/08838151.2017.1309410

- LARRAUFIE, A. \& SOMMER, F. (2015). Motivations behind becoming a blogger and their business implications: The case of fashion. En S. RATHORE \& A. PANWAR (Eds.), Capturing, Analyzing, and Managing Word-of-Mouth in the Digital Marketplace (pp. 103-118). Hershey: IGI Global.

- LAZARSFELD, P., BERELSON, B. \& GAUDET, H. (1968). The People's Choice: How the Voter Makes up His Mind in a Presidential Campaign. Nueva York: Columbia University Press.

- LEE, H. (2016). Making of celebrities: A comparative analysis of Taiwanese and American fashion bloggers. Advances in Consumer Research, 44, 319-323.

- MARTÍN GARCÍA, T. \& MARTÍNEZ SOLANA, Y. (2017). Blogs de moda: Nuevos preservadores de los estereotipos de género de las revistas femeninas. En J.C. SUÁREZ; L.A. GUADARRAMA; J. VALERO \& P. PANARESE (Eds.), La desigualdad de género invisibilizada en la comunicación (ebook). Madrid: Dykinson.

- MORA, C. (2005, Junio, 16). Cuánto cuesta un desfile. Yo Dona, 18, 50-55.

- MORTARA, A. \& ROBERTI, G. (2017). The Spread Fashion: an Explorative Research of Italian Fashion Blog. Italian Sociological Review, 7(1), 87-104. doi: 10.13136/isr.v7i1.150

- OVELAR, M. (2017, 16 de septiembre). Así funciona el lucrativo negocio de ser 'influencer' en España. El País. Recuperado de: https://smoda.elpais.com/ moda/moda-influencers/

- PARGA, M. (2015, 22 de abril). ¿Qué queda de los blogs de moda?. Vogue. Recuperado de https://goo.gl/C9psEv

- PERTHUIS, K. (2016). People in Fashionable Clothes: Street Style Blogs and the Ontology of the Fashion Photograph. Fashion Theory - Journal of Dress Body and Culture, 20(5), 523-543. doi: 10.1080/1362704X.2015.1115656 
- PIHL, C. \& SANDSTRÖM, C. (2013). Value creation and appropriation in social media: The case of fashion bloggers in Sweden. International Journal of Technology Management, 61(3/4), 309-323. doi: 10.1504/IJTM.2013.052673

- RAMOS-SERRANO, M. \& JIMÉNEZ-MARÍN, G. (2014). Blogs y Moda: cuando los usuarios crean las tendencias. En D. FERNÁNDEZ \& M. RAMOS-SERRANO (Eds), Tecnologías de la persuasión: uso de las TIC en publicidad y relaciones públicas (pp. 131-143). Barcelona: UOC.

- RUIZ MOLINA, E. (2013). Los blogs de moda en España: De la espontaneidad del usuario a la profesión de blogger. RUTA Comunicación, 5, 1-25. Recuperado de https://goo.gl/XAoGHi

- STRÄHLE, J. \& GRÜNEWALD, A.K. (2017). The prosumer concept in fashion retail: Potentials and Limitations. En J. STRÄHLE (Ed.), Green fashion retail (pp. 95-119). Singapur: Springer.

- TitTon, M. (2015). Fashionable Personae: Self-identity and Enactments of Fashion Narratives in Fashion Blogs. Fashion Theory, 19(2), 201-220. doi: 10.275 2/175174115X14168357992391

- TOFFLER, A. (1980). La tercera ola. Bogotá: Editorial Plaza \& Janes.

- UZUNOGLU, E. \& MISCI, S. (2014). Brand communication through digital influencers: Leveraging blogger engagement. International Journal of Information Management; 34, 552-602. doi: 10.1016/j.ijinfomgt.2014.04.007

- WIAŻEWICZ, J. \& ZATWARNICKA-MADURA, B. (2016). Fashion blogs as the information sources about trends and new products in fashion management. Polish Journal of Management Studies, 14(1), 214-225. doi: 10.17512/ pjms.2016.14.1.20 


\section{Biografías}

\section{Laura Esteban Santos}

Universidad de Valladolid, España

laura.esteban000@gmail.com

Graduada en Marketing e Investigación de Mercados por la Universidad de Valladolid, donde obtuvo el Premio Extraordinario. Posee un Máster en International Fashion Marketing por la Glasgow Caledonian University (Reino Unido). Es miembro del Ilustre Colegio de Economistas de Valladolid y ha realizado diversas investigaciones relativas a los blogs de moda, así como también ha colaborado con negocios en su desarrollo de marketing digital.

\section{Irene García-Medina}

Glasgow Caledonian University, Reino Unido

Irene.Garcia2@gcu.ac.uk

Doctora en Marketing (University of Sophia, Antipolis, France) $\mathrm{y}$ en International Relations (University of Vienna, Austria), es profesora de Marketing en la Glasgow Caledonian University (Reino Unido). Ha dado ponencias sobre marketing en varios países y ha publicado numerosos artículos. Es miembro desde 2008 del comité internacional M-LIFE Conference and Exhibitions, Mobile Government Consortium International (Brighton, Reino Unido), así como también es miembro del comité editorial de las revistas Aprendizaje 21 y Obra Digital. 


\section{Elena Bellido-Pérez}

Universidad de Sevilla, España

ebellido@us.es

Contratada pre-doctoral en el Departamento de Comunicación Audiovisual y Publicidad de la Universidad de Sevilla, donde realiza su tesis doctoral sobre arte y propaganda dentro del Programa de Doctorado Interuniversitario en Comunicación. Graduada en Publicidad y Relaciones Públicas (Premio Extraordinario Fin de Estudios) y Máster en Comunicación y Cultura. Compagina la docencia con la investigación en líneas temáticas concretas como la comunicación digital, el arte, la propaganda o la ideología. 


\section{Anexo}

\section{Cuestionario distribuido a través de Google Forms:}

1. ¿Sigues habitualmente algún blog de moda?

Sí
No

2A. (Seguidoras) Indica un bloguero/a de moda que conozcas y sigas en la actualidad / (No seguidoras) Indica un bloguero/a de moda que conozcas (en el caso de que conozcas alguno)

2B. (Seguidoras) Es el bloguero/a que más sigo porque...

\begin{tabular}{|c|c|c|c|c|c|}
\hline & $\begin{array}{c}1 . \\
\text { Totalmente } \\
\text { en } \\
\text { desacuerdo }\end{array}$ & 2 & 3 & 4 & $\begin{array}{l}5 . \\
\text { Totalmente } \\
\text { de acuerdo }\end{array}$ \\
\hline ...me gusta su estilo & O & 0 & 0 & 0 & O \\
\hline ...me interesa su vida & O & 0 & O & 0 & O \\
\hline ...me identifico con él/ella & O & O & O & O & O \\
\hline $\begin{array}{c}\text {...es el/la que está más de } \\
\text { moda }\end{array}$ & 0 & 0 & 0 & $\mathrm{O}$ & $\mathrm{O}$ \\
\hline ...lo siguen mis amigos & $\mathrm{O}$ & $\mathrm{O}$ & $\mathrm{O}$ & $\mathrm{O}$ & O \\
\hline ...me inspira & $\mathrm{O}$ & $\mathrm{O}$ & $\mathrm{O}$ & $\mathrm{O}$ & $\mathrm{O}$ \\
\hline ...es el más entretenido & O & $\mathrm{O}$ & O & $\mathrm{O}$ & $\mathrm{O}$ \\
\hline ...me gustan sus fotos & $\mathrm{O}$ & $\mathrm{O}$ & $\mathrm{O}$ & $\mathrm{O}$ & O \\
\hline ...aprendo sobre moda & $\mathrm{O}$ & $\mathrm{O}$ & O & $\mathrm{O}$ & $\mathrm{O}$ \\
\hline $\begin{array}{l}\text {...me fío de la } \\
\text { calidad/autenticidad de la } \\
\text { información }\end{array}$ & 0 & 0 & 0 & 0 & 0 \\
\hline $\begin{array}{c}\text {...creo que actúa de buena } \\
\text { fe }\end{array}$ & 0 & 0 & 0 & 0 & 0 \\
\hline $\begin{array}{l}\text {...creo que es conocedor de } \\
\text { los temas que trata }\end{array}$ & O & $\mathrm{O}$ & $\mathrm{O}$ & 0 & 0 \\
\hline
\end{tabular}


2C. (Seguidoras) Le/la sigo en... / (No seguidoras) Le/la conozco por...

Su blog

Instagram

Ambos

2D. (Seguidoras) ¿Con qué frecuencia visitas su blog?

Nunca

De forma muy esporádica

Al menos una vez en semana

Todos los días

2E. (Seguidoras) Marca la opción que más se ajuste a ti en las siguientes cuestiones sobre el seguimiento de tu bloguero/a favorito/a

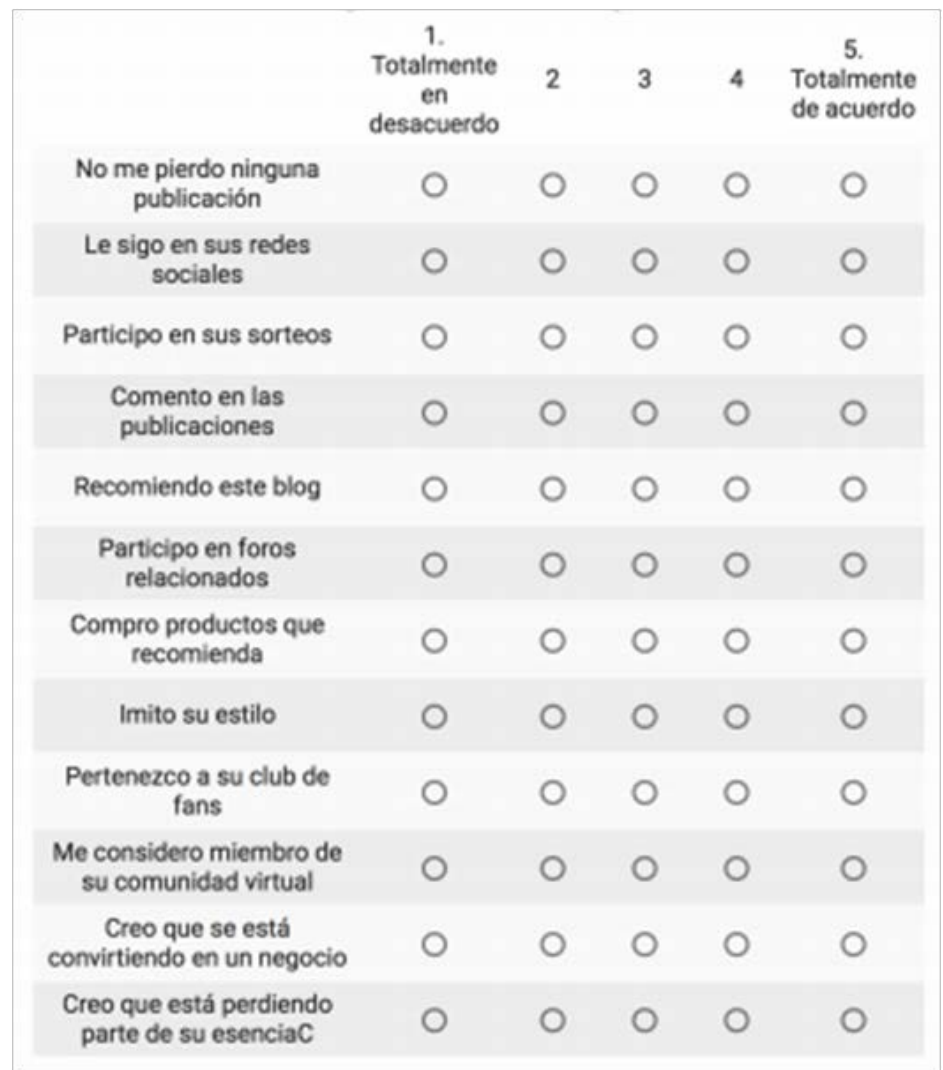


3. (Seguidoras) Cuando visitas un blog de moda, ¿en qué grado prefieres cada uno de los siguientes tipos de publicaciones? / (No seguidoras). En caso de que decidieras seguir algún blog de moda, ¿cuál crees que sería tu grado de preferencia para cada uno de los siguientes tipos de publicaciones?

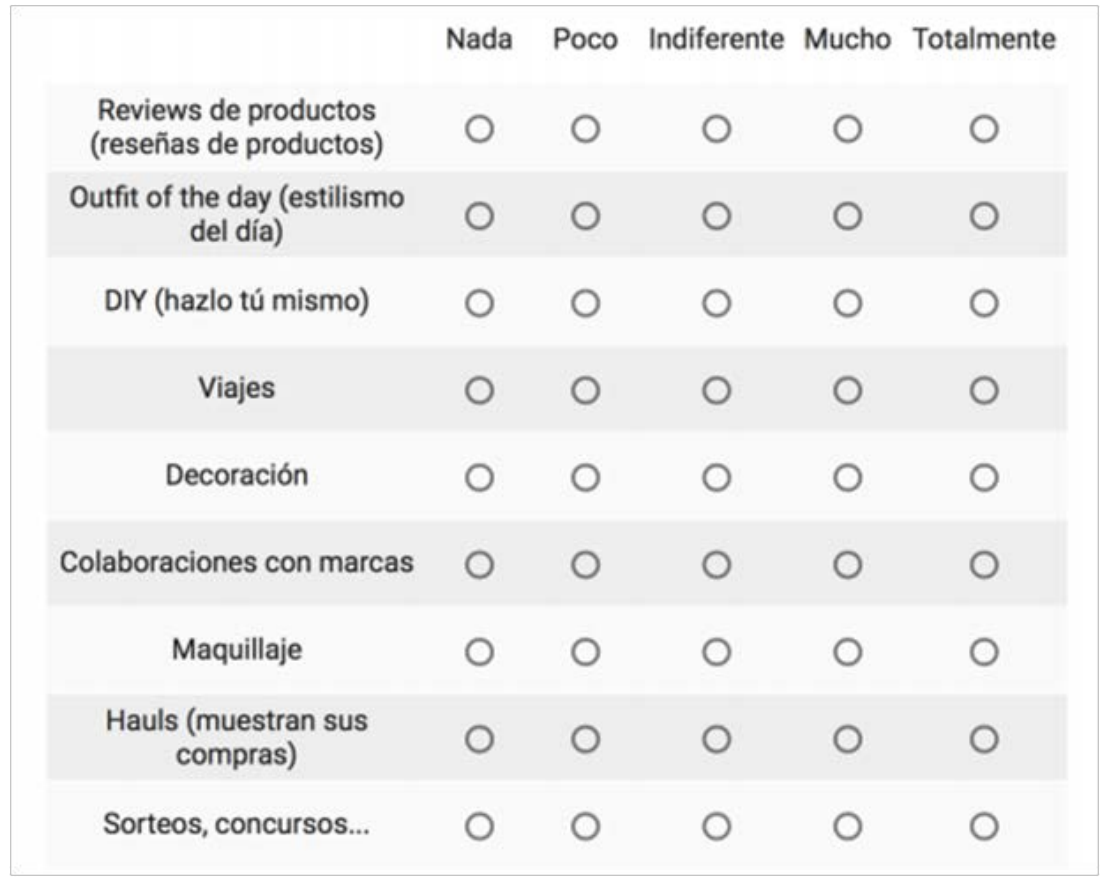

4. (Seguidoras) ¿En qué medida crees que te influyen cada uno de los siguientes aspectos a la hora de seguir un blog o no?

\begin{tabular}{ccccccc}
\hline & Nada & Poco & Bastante & Mucho & Totalmente \\
Entorno (amigos, familia...) & 0 & 0 & 0 & 0 & 0 \\
Soy fan de la persona & 0 & 0 & 0 & 0 & 0 \\
Cercanía del bloguero & 0 & 0 & 0 & 0 & 0 \\
Otras Redes Sociales & 0 & 0 & 0 & 0 & 0 \\
$\begin{array}{c}\text { Revistas } \\
\text { estilo de vida... }\end{array}$ & 0 & 0 & 0 & 0 & 0 \\
Identificación con el bloguero & 0 & 0 & 0 & 0 & 0 \\
Credibilidad del blog & 0 & 0 & 0 & 0 & 0 \\
Accesibilidad del blog & 0 & 0 & 0 & 0 & 0 \\
\hline
\end{tabular}


5. (Seguidoras y no seguidoras) A la hora de buscar inspiración para tus compras, ¿consultas blogs de moda?

Sí

No

6. (Seguidoras) De los productos que se muestran en el blog, ¿cuántos sueles comprar?

Prácticamente ninguno

Bastantes

Muchos

Casi todos 